\title{
Fuzzy Evaluation on University Student Aerobics Club Education Mode
}

\author{
Jinxuan Huang*
}

Sports Work Department, Civil Aviation University of China, Tianjin 300300, China

\begin{abstract}
Sports in the form of clubs have a long history in foreign countries, such as Japan that started later, its club sports management and operation have gained development to higher level in short 40 years, while in some western world, sports in the form of clubs even is supported by the mass, and the clubs are also the places that sports activities most frequently appear, for mass residents, the emergence of club sports becomes the main form in bodybuilding. The paper evaluates theory of learning, technology assessment, basic ability of assessment and the self evaluation, it gets the weight result $z=\left(\begin{array}{llll}0.3 & 0.4 & 0.25 & 0.05\end{array}\right)$, which shows during university student aerobics sports assessment mode, technology assessment occupied $40 \%$ of the assessment mode, theory of learning occupies $30 \%$, basic ability of assessment occupies $25 \%$, and the self evaluation occupies $5 \%$.
\end{abstract}

Keywords: Aerobics, Club, Assessment mode, Fuzzy Comprehensive evaluation, Fitness effects.

\section{INTRODUCTION}

Aerobics originated from America, it went abroad and developed around the world in the beginning of eighties, 20th century [1]. Then, Britain firstly founded aerobics association in 1984, and subsequently it became a country that owned the largest aerobics organizations in Europe [2]. Driven by British aerobics organizations, aerobics gained rapidly development in other countries of Europe [3].

Since China entering into 21 st century, comprehensive national power has constantly risen, people's material and spiritual pursuits have also increasingly strengthened; therefore most of people have begun to have the enthusiasm to participate in all kinds of sports events. However, aerobics as a kind of sports that can build up one's body and also cultivate one's taste of these events, it becomes a favorite sport in public, and also due to its good effects in weight losing, is favored by women [4].

As a new style sport event, Chinese aerobics has gained rapidly development with the help of Chinese aerobics rhythmic gymnastics association from 2000 to 2005 due to being loved by teenagers and university students.

\section{MODEL ESTABLISHMENTS}

Utilize fuzzy comprehensive evaluation, steps are as following:

(1) Establish factor set $U: U=\left(\begin{array}{llll}U_{1} & U_{2} & \cdots & U_{k}\end{array}\right)$

(2) Establish judgment set $V$ ( evaluation set), $V=\left(\begin{array}{llll}V_{1} & V_{2} & \cdots & V_{n}\end{array}\right)$
According to general evaluation system, define evaluation grade domain:

$V=\left\{V_{1}, V_{2}, V_{3}, V_{4}\right\}=\{$ Very goodgood, normal, bad $\}$

(3) Establish judgment matrix fuzzy mapping from $U$ to $V$, it gets fuzzy relation as following matrix shows:

$$
R=\left[\begin{array}{cccc}
r_{11} & r_{12} & \cdots & r_{1 n} \\
r_{21} & r_{22} & \cdots & r_{2 n} \\
\vdots & \vdots & & \vdots \\
r_{m 1} & r_{m 2} & \cdots & r_{m n}
\end{array}\right]
$$

(4) Establish weight set, $A=\left(a_{1}, a_{2}, \cdots, a_{n}\right)$, it meets conditions: $\sum_{i=1}^{n} a_{i}=1 \quad a_{i} \geq 0$

(5) Fuzzy relation $R$ every line reflects the line influence factors to object judgment extent, and meanwhile, $R$ every column reflects the column influence factors to object judgment extent [5].

$$
\begin{aligned}
& \sum_{i=1}^{n} r_{i j} \quad j=1,2,3, \cdots, m \\
& B=A \cdot R \\
& \quad=\left(a_{1}, a_{2}, a_{3}, \cdots, a_{n}\right) \cdot\left[\begin{array}{cccc}
r_{11} & r_{12} & \cdots & r_{1 n} \\
r_{21} & r_{22} & \cdots & r_{2 n} \\
\vdots & \vdots & & \vdots \\
r_{m 1} & r_{m 2} & \cdots & r_{m n}
\end{array}\right] \\
& \quad=\left(b_{1}, b_{2}, b_{3}, \cdots, b_{n}\right)
\end{aligned}
$$

In $V$, fuzzy combination is evaluation set $B$. Based on above described facts, actual change model is: 


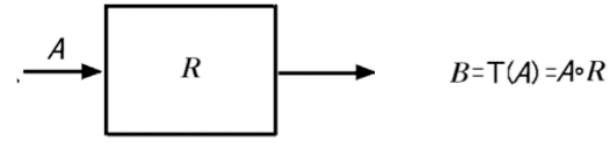

Fig. (1). Changed model.

As Fig. (1) show, it gets fuzzy comprehensive evaluation changed model, and can establish corresponding every factor grade evaluation transformation function, evaluation factors $\mathrm{u} 1, \mathrm{u} 2, \mathrm{u} 3, \mathrm{u} 4, \mathrm{u} 5$ membership functions can be expressed as following:

$$
\begin{aligned}
& u_{v 1}\left(u_{1}\right)=\left\{\begin{array}{cc}
0.5\left(1+\frac{u_{i}-k_{1}}{u_{i}-k_{2}}\right), & u_{i} \geq k_{1} \\
0.5\left(1-\frac{k_{1}-u_{i}}{k_{1}-k_{2}}\right), & k_{2} \leq u_{i}<k_{1} \\
0 &
\end{array}\right. \\
& 0 \quad, \quad u_{i}<k_{2} \\
& u_{v 2}\left(u_{1}\right)=\left\{\begin{array}{lr}
0.5\left(1-\frac{u_{i}-k_{1}}{u_{i}-k_{2}}\right), & u_{i} \geq k_{1} \\
0.5\left(1+\frac{k_{1}-u_{i}}{k_{1}-k_{2}}\right), & k_{2} \leq u_{i}<k_{1} \\
0.5\left(1-\frac{u_{i}-k_{3}}{k_{2}-k_{3}}\right), & k_{3} \leq u_{i}<k_{2} \\
0.5\left(1-\frac{k_{3}-u_{i}}{k_{2}-u_{i}}\right), & u_{i}<k_{3}
\end{array}\right. \\
& u_{v 1}\left(u_{1}\right)=\left\{\begin{array}{cc}
0, & u_{i} \geq k_{2} \\
0.5\left(1-\frac{k_{1}-u_{i}}{k_{2}-k_{3}}\right), & k_{3} \leq u_{i}<k_{2} \\
0.5\left(1+\frac{k_{3}-u_{i}}{k_{2}-u_{i}}\right), & u_{i}<k_{3}
\end{array}\right.
\end{aligned}
$$

\section{Combine with Fuzzy Evaluation Model to Evaluate Aerobics Education Mode}

It gets aerobics assessment contents evaluation structure chart, it shows first grade assessment indicators and second grade assessment indicators in (Fig. 2).

Establish factor set $U, U=\left(\begin{array}{llll}U_{1} & U_{2} & U_{3} & U_{4}\end{array}\right)$. Among them, theory of learning is $U_{1}$, technology assessment is $U_{2}$, basic ability of assessment is $U_{3}$, the self evaluation is $U_{4}$, it gets Table 1.

By Table 1 listed factors, it gets evaluation sets.

$$
\begin{aligned}
& U_{1}=\left\{u_{11}, u_{12}\right\} ; U_{2}=\left\{u_{21}, u_{22}\right\} \\
& U_{3}=\left\{u_{31}, u_{32}, u_{33}\right\} ; U_{4}=\left\{u_{41}, u_{42}, u_{43}, u_{44}\right\}
\end{aligned}
$$

By collecting data and analyzing, it gets theory of learning $U_{1}$, technology assessment $U_{2}$, basic ability of assessment $U_{3}$, the self evaluation $U_{4}$ four kinds of factors importance degrees ranking statistics, as Table $\mathbf{2}$ shows.

By Table 2 sorting, it gets theory of learning $U_{1}$, technology assessment $U_{2}$, basic ability of assessment $U_{3}$, the self evaluation $U_{4}$ four aspects ranking matrixes:

$$
\begin{aligned}
& U_{1}=\{23,7,3,0\}, U_{2}=\{7,18,80\} \\
& U_{3}=\{0,9,13,12\}, U_{4}=\{3,0,9,21\}
\end{aligned}
$$

Obtained weighted vector from rank 1 to rank 2:

$\beta=\left\{\beta_{1}, \beta_{2}, \beta_{3}, \beta_{4}\right\}=\{0.4,0.3,0.2,0.1\}$

$U_{i}^{*}=U_{i} \cdot \beta^{T}$

$U_{1}^{*}=12, U_{2}^{*}=9.7, U_{3}^{*}=6, U_{4}^{*}=5$

The paper takes normalization processing:

$U_{1}^{*}=0.35, U_{2}^{*}=0.3, U_{3}^{*}=0.2, U_{4}^{*}=0.15$

It gets: $\bar{A}=\left(\begin{array}{llll}0.35 & 0.3 & 0.2 & 0.15\end{array}\right)$

Through aerobics assessment reflection, the paper gets remarks membership as Table $\mathbf{3}$ shows.

By one aerobics assessment mode each indicator obtained evaluation, the paper gets Table 4.

By above model, it gets single layer indicator weight factor fuzzy set is:

$U_{1}^{*}=\left\{U_{11}, U_{12}, U_{13}, U_{14}, U_{15}\right\}=\left\{\begin{array}{llll}0.25 & 0.250 .2 & 0.15 & 0.15\end{array}\right\}$

$U_{2}^{*}=\left\{U_{21}, U_{22}, U_{23}, U_{24}\right\}=\left\{\begin{array}{llll}0.54 & 0.10 .24 & 0.14\end{array}\right\}$

$U_{3}^{*}=\left\{U_{31}, U_{32}, U_{33}, U_{34}\right\}=\left\{\begin{array}{llll}0.4 & 0.3 & 0.1 & 0.2\end{array}\right\}$

$U_{4}^{*}=\left\{U_{41}, U_{42}, U_{43}\right\}=\{0.30 .40 .3\}$

By Table 4, and combine with Table 3 remarks membership, the paper gets theory of learning $U_{1}$, technology assessment $U_{2}$, basic ability of assessment $U_{3}$, the self evaluation $U_{4}$ each aspect evaluation set:

Theory of learning $U_{1}=\left(\begin{array}{cccc}0 & 0 & 0.05 & 0.95 \\ 0 & 0 & 0.05 & 0.95\end{array}\right)$

Technology assessment $U_{2}=\left(\begin{array}{cccc}0 & 0 & 0.05 & 0.95 \\ 0 & 0 & 0.05 & 0.95\end{array}\right)$

Basic ability of assessment $U_{3}=\left(\begin{array}{cccc}0 & 0 & 0.05 & 0.95 \\ 0 & 0.05 & 0.9 & 0.05 \\ 0 & 0.05 & 0.9 & 0.05\end{array}\right)$

The self evaluation $U_{4}=\left(\begin{array}{cccc}0 & 0 & 0.05 & 0.95 \\ 0 & 0.05 & 0.9 & 0.05 \\ 0 & 0.05 & 0.9 & 0.05 \\ 0.05 & 0.9 & 0.05 & 0\end{array}\right)$

$B_{i}=A_{i} \cdot R_{i}$

Make normalization processing with obtained $B_{i}$, it gets fuzzy evaluation matrix. 


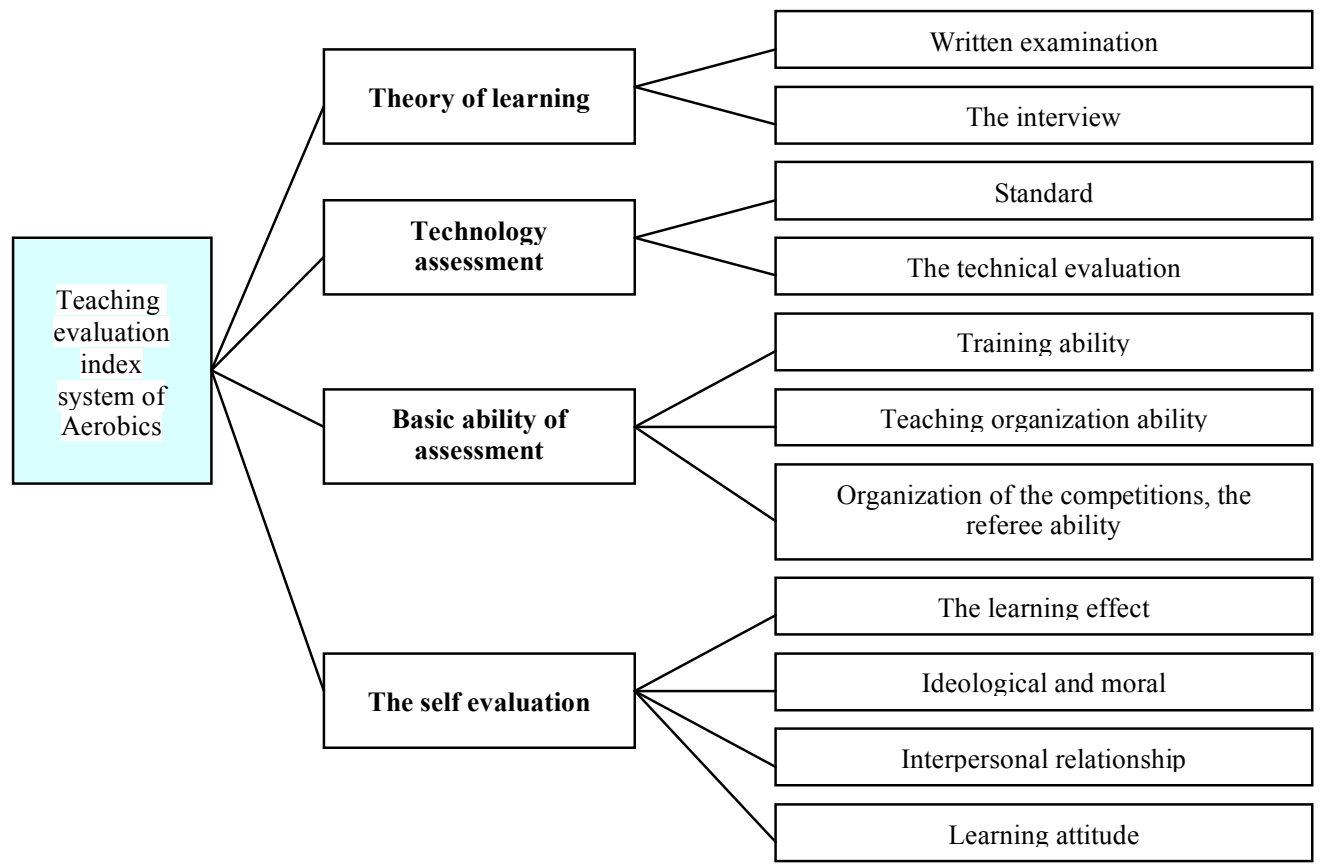

Fig. (2). Aerobics assessment contents evaluation structure chart.

Table 1. Aerobics education evaluation index system.

\begin{tabular}{|c|c|c|c|}
\hline Theory of learning $U_{1}$ & Technology assessment $U_{2}$ & Basic ability of assessment $U_{3}$ & The self evaluation $U_{4}$ \\
\hline Oral examination $u_{11}$ & Reach the standard $u_{21}$ & Teaching organizational ability $u_{31}$ & Ideology and morality $u_{41}$ \\
\hline Written examination $u_{12}$ & Assessment of technique $u_{22}$ & Exercise training capacity $u_{32}$ & Learning effect $u_{42}$ \\
\hline & & $\begin{array}{c}\text { Competition organization, } \\
\text { judgment capacity } u_{33}\end{array}$ \\
\hline
\end{tabular}

Table 2. Four kinds of factors importance degree ranking statistics.

\begin{tabular}{|c|c|c|c|}
\hline Classification & Rank 1 & Rank 2 & Rank 4 \\
\hline \hline Theory of learning $U_{1}$ & 23 & 7 & 3 \\
\hline Technology assessment $U_{2}$ & 7 & 9 & 8 \\
\hline Basic ability of assessment $U_{3}$ & 0 & 0 & 13 \\
\hline The self evaluation $U_{4}$ & 3 & 9 & 21 \\
\hline
\end{tabular}

$\bar{B}=\left(\begin{array}{l}B_{1} \\ B_{2} \\ B_{3} \\ B_{4}\end{array}\right)=\left(\begin{array}{cccc}0.07 & 0.27 & 0.13 & 0.53 \\ 0 & 0.1 & 0.4 & 0.5 \\ 0.08 & 0.46 & 0.38 & 0.08 \\ 0.14 & 0.2 & 0.3 & 0.36\end{array}\right)$

It gets comprehensive evaluation value: $D=U^{*} \cdot B=\left(\begin{array}{llll}0.3 & 0.4 & 0.25 & 0.05\end{array}\right)$

\section{CONCLUSION}

Up to now, development of fuzzy mathematics has 50 years history, though it is a relative new discipline, it has more plentiful contents in theory, and it gets involved in multiple disciplines. Evaluation is a kind of human thinking process, but it is not linear changing.

Based on fuzzy mathematics characteristics, the paper analyzes university aerobics education mode through fuzzy mathematics. For aerobics course assessment mode, it needs to consider multiple influence factors, as theory of learning, technology assessment, basic ability of assessment and the self evaluation. It gets the weight result $D=\left(\begin{array}{llll}0.3 & 0.4 & 0.25 & 0.05\end{array}\right)$.

It shows during university aerobics sports assessment mode, technology assessment occupied $40 \%$ of the assessment 
Table 3. Remarks membership.

\begin{tabular}{|c|c|c|c|c|}
\hline \multirow{2}{*}{ Evaluation way } & \multicolumn{5}{|c|}{ Set Scores Interval } \\
\cline { 2 - 5 } & $\mathbf{0 - 6 0}$ & $\mathbf{6 0 - 8 0}$ & $\mathbf{8 0 - 9 0}$ & $\mathbf{9 0 - 1 0 0}$ \\
\hline \hline Very good & 0 & 0 & 0.05 & 0.95 \\
\hline Good & 0 & 0.05 & 0.9 & 0.05 \\
\hline Normal & 0.05 & 0.9 & 0 & 0 \\
\hline Bad & 0.95 & 0.05 & 0.05 & 0 \\
\hline
\end{tabular}

Table 4. Aerobics assessment mode each indicator obtained evaluation value.

\begin{tabular}{|c|c|c|c|}
\hline Each Layer Indicator & Evaluation Value & Each Layer Indicator & Evaluation Value \\
\hline \hline Oral examination $u_{11}$ & Normal & Ideology and morality $u_{41}$ & Very good \\
\hline Written examination $u_{12}$ & Normal & Learning effect $u_{42}$ & Good \\
\hline Reach the standard $u_{21}$ & Good & Interpersonal relationship $u_{44}$ & Normal \\
\hline Assessment of technique $u_{22}$ & Very good & \\
\hline Teaching organizational ability $u_{31}$ & Normal & & \\
\hline Exercise training capacity $u_{32}$ & Very good & & \\
\hline
\end{tabular}

mode, theory of learning occupies $30 \%$, basic ability of assessment occupies $25 \%$, and the self evaluation occupies $5 \%$.

\section{CONFLICT OF INTEREST}

The author confirms that this article content has no conflict of interest.

\section{ACKNOWLEDGEMENTS}

Declared none.

\section{REFERENCES}

[1] W. Gong, Y. Xu, and H. Hu, "Universities public physical education health education course innovation system study and practice," Journal of Wuhan Institute of Physical Education, vol. 4, no.1, pp. 119-123, 2004.

[2] P-W. Han, Y. Wang, "Discuss on public aerobics special effects on Chinese national fitness exercises," Journal of Xi'an Physical Education University, vol. 22, no. 6, pp. 22-23, 1998.

[3] Z. Li, "Fujian province universities aerobics teams current situation analysis and development measures," Journal of Jilin Physical Culture Institute, vol. 30, no. 2, pp. 117-123, 2006.

[4] P. Xu, "Sports education under humanistic education philosophy and new situation-briefly discussion sports teachers role positioning under new curriculum reform situation," Physical Education, vol. 51, no. 4, pp. 32-39, 2006.

[5] T. Zhang, B. Jiang, X. Gu, "Research on university sports education mode," Sports Science, vol. 20, no. 3, pp. 29-31, 2003.

(C) Jinxuan Huang; Licensee Bentham Open.

This is an open access article licensed under the terms of the (https://creativecommons.org/licenses/by/4.0/legalcode), which permits unrestricted, noncommercial use, distribution and reproduction in any medium, provided the work is properly cited. 